\title{
Serratus anterior plane block for minimal invasive heart surgery
}

Vivien Berthoud ${ }^{1 *}$ (D, Omar Ellouze', Maxime Nguyen ${ }^{1}$, Maria Konstantinou', Serge Aho², Ghislain Malapert ${ }^{3}$, Claude Girard ${ }^{1}$, Pierre-Gregoire Guinot ${ }^{1}$, Olivier Bouchot ${ }^{3}$ and Belaid Bouhemad ${ }^{1}$

\begin{abstract}
Background: Minimal invasive heart surgery (MIHS) presents several benefits, but provides intense and prolonged post-operative pain. Our objective was to compare efficacy of serratus anterior plane block (SAPB) with continuous wound infiltration (CWI) for management of post-operative pain following MIHS.

Methods: It's retrospective, monocentric study between November 2016 to April 2017. The study was performed at the University hospital of Dijon, Burgundy, France. All patients scheduled for MIHS was included. Data was collected retrospectively. During this period, 20 patients had SAPB and 26 had CWI. SAPB was performed before extubation with a single injection of $0.5 \mathrm{mg} / \mathrm{kg}$ of ropivacaine $(5 \mathrm{mg} / \mathrm{ml})$. In the CWI group, catheter was inserted in the subcutaneous space by the surgeon at the end of the procedure. A $10 \mathrm{ml}$ bolus of ropivacaine $(7.5 \mathrm{mg} / \mathrm{mL})$ was followed by a continuous infusion ( $2 \mathrm{mg} / \mathrm{ml}$ ) between 7 and $12 \mathrm{ml} / \mathrm{h}$ for $48 \mathrm{~h}$. Morphine consumption and visual analog score (VAS) were recorded for $48 \mathrm{~h}$. Length of stay in intensive care unit and hospital was also collected.
\end{abstract}

Results: Morphine consumption and VAS score were significantly lower in SAPB group $(p<0.01)$. Length of stay in intensive care and hospital was significantly was decreased in SAPB group.

Conclusion: SAPB appears effective in reducing postoperative MIHS pain.

Keywords: Serratus anterior plane block, Pain, Post operative, Minimal invasive heart surgery, Thoracotomy

\section{Background}

Minimally-invasive heart surgery (MIHS) for aortic, mitral valve replacement or repair has developed rapidly. It presents several advantages including decreasing the risk of mediastinitis, leaving a more esthetic scar, facilitating post-operative rehabilitation and reducing hospital length of stay, compared with sternotomy [1]. MIHS requires thoracic incision in the right 4th or 5th intercostal space and is followed by intense and prolonged post-operative pain $[2,3]$. The pain is exacerbated by breathing movements, coughing and respiratory physiotherapy. It is most severe during the first $48 \mathrm{~h}$ after surgery with repercussions on breathing, cognitive, urinary and digestive function, and may prevent early rehabilitation.

Usually, pain is treated with morphine agonist, which also have many side effects, affecting the duration of

\footnotetext{
*Correspondence: vivien.berthoud@chu-dijon.fr

'Unité d'Anesthesie Réanimation Cardio-Vasculaire, CHU François Mitterrand, BP 77908, 21709 Dijon Cedex, France

Full list of author information is available at the end of the article
}

post-operative sedation, hemodynamic stability and respiratory function, particularly in elderly patients $[4,5]$.

The management of pain requires a multimodal approach that associates neuraxial anesthesia (spinal or thoracic epidural anesthesia), systemic opiates and step I or II analgesics [6,7]. The use of the perimedullary technique for analgesia in cardiac surgery is limited by the fear of perimedullary hematoma $[8,9]$. Continuous wound infiltration (CWI) analgesia is an effective practice for management of postoperative pain in a multimodal approach, especially during thoracotomy, but which is probably still insufficient [10].

Serratus anterior plane block (SAPB) is a recently described technique for chest wall analgesia. SAPB provide anesthesia of dermatomes $\mathrm{T} 2$ to $\mathrm{T} 9$, where surgical incision is realized (4th or 5th right intercostal space). The procedure consists of injecting local anesthetic between the muscle planes of the chest wall to block the lateral cutaneous branches of the intercostal nerves [11]. SAPB has already shown its efficacy in the management of 
post-operative pain following breast surgery, thoracoscopy, thoracotomy and multiple rib fractures [12-16]. No data has yet been published on the effectiveness of analgesia by SAPB for MIHS.

Several loco-regional analgesic techniques are available for postoperative analgesia of thoracotomy. Currently, in minimally invasive cardiac surgery no study has compared CWI c SAPB. Because the serratus block allows extensive analgesia, our hypothesis is that SAPB is superior to CWI in terms of analgesia. Our objective was to demonstrate the superiority of SAPB compared to CWI in the management of post-operative pain following MIHS.

\section{Methods}

\section{Study design}

We performed a retrospective analysis of the data of consecutive patients operated from November 2016 to April 2017 at our institution. Before implementing this new technique routinely and analyzing our data on these patients, we performed SABP with setting systematically a CWI and a morphine patient controlled analgesia in case of inefficiency of the SABP. Decision to realize SABP was done in agreement with the surgical team and some of the anesthesiologists who realize ultrasound guided regional anesthesia. Accordance to french law, an ethical committee is not necessary to realize a new locoregional analgesia technique, and this is part of the professional practices prerogatives. Nevertheless, we systematically inform all patients of the postoperative analgesia technique that will be performed, including this new technique (SABP) during preoperative anesthesia consultation, but no written consent was collected. Because, the study is a retrospective analysis of institutional data, and in compliance with the French law on clinical research, we only had to obtain French personal data protection authorization. We submitted our study to the national commission for data protection (CNIL) and obtained authorization 2,053,943 v 0 . The present report was drafted in line with the Strengthening Reporting of Observational Studies in Epidemiology statement for cohort studies.

The inclusion criteria were patients who underwent MISH. Exclusion criteria were: patients under 18 years old, pregnancy, allergies to local anesthesics, cognitive dysfunction, and post-operative ventilation during $\geq 24 \mathrm{~h}$.

\section{Anesthesia protocol}

In our unit, anesthesic and post-operative management is protocolized. Anesthesia consultation was carried out at least $48 \mathrm{~h}$ before the intervention, and premedication consisted of oral gabapentin $300 \mathrm{mg}$ the day before and the morning of the surgery. Anesthesia was given accordingly to protocol in use. After pre-oxygenation with 100\% inspired oxygen, anesthesia was performed with propofol, sufentanil and cisatracurium for obtained bispectral index between 60 to 40 and neuromuscular transmission $(\mathrm{NMT})=0 / 4$. Orotracheal intubation (OTI) was performed with a double-lumen endotracheal tube. Post-operative pain was managed by continuous CWI or SAPB. Group selection was based on the anesthetist in charge of the patient and familiar with SABP.

The CWI catheter was inserted into the subcutaneous space by the surgeon at the end of the intervention. The catheter was purged with a bolus of $10 \mathrm{ml}$ of ropivacaine at $7.5 \mathrm{mg} / \mathrm{ml}$. An elastomeric pump containing $300 \mathrm{ml}$ of ropivacaïne at $2 \mathrm{mg} / \mathrm{ml}$ was set up allowing a continuous infusion at $7 \mathrm{~mL} / \mathrm{h}$ for $48 \mathrm{~h}$. The flow rate was increased up to $12 \mathrm{ml} / \mathrm{h}$ if the pain NRS was $>3$.

The SAPB was performed by the anesthetist (medical team) at the end of the surgery. Ultrasonography was used with the transducer placed against the midaxillary line to visualize the pleura, the lateral arch of the 4th or 5 th rib and the different muscle planes from the most superficial to the deepest: the latissimus dorsi muscle, the serratus anterior muscle and the intercostal muscles. The needle (VYGON locoplex 50 or $100 \mathrm{~mm}$ ) was inserted in the plane of the transducer into the 4th or 5th intercostal space, the progress of the needle was followed on the screen until it reached the space between the serratus anterior muscle and the intercostal muscles. A single injection of $0.5 \mathrm{ml} / \mathrm{kg}$ of ropivacaine $5 \mathrm{mg} / \mathrm{ml}$ was given without exceeding a maximal dose of $200 \mathrm{mg}$. During the injection, we looked for the formation of a bi-convex lens shape between the two muscles (Fig. 1).

At the end of surgery, all patients were sedated with propofol and the lungs were mechanically ventilated until haemodynamic stability and normothermia were obtained and blood loss was considered acceptable (less than $1 \mathrm{ml} \mathrm{kg}^{-1} \mathrm{~h}^{-1}$ ). After obtaining the 3 criteria above, extubation is carried out according to the protocol of the service. Every patient had an intravenous titration of morphine with a bolus of 2 to $3 \mathrm{mg}$ every $7 \mathrm{~min}$ from extubation, until a pain visual analogue scale (NRS) below 3 was achieved. Then PCA morphine was set up with the following parameters: $1 \mathrm{mg}$ bolus, refractory period of $7 \mathrm{~min}$, maximum dose of $20 \mathrm{mg}$ every $4 \mathrm{~h}$ and without continuous infusion. In addition, the patients were systematically given $1 \mathrm{~g}$ of intravenous paracetamol every $6 \mathrm{~h}$ on day 0 and then orally from day 1 . The use of other analgesics if the pain NRS remained above 3 was left to the discretion of the physician.

The following data we retrieved from the data base: morphine consumption, at NRS h0, h6, h24, use of additional analgesics (ketoprofen, tramadol or nefopan), nausea and vomiting episode, need for non-invasive ventilation (NIV), during the first $48 \mathrm{~h}$ after surgery. We also recorded length of ICU and hospital stay. All study data are available in an additional file attached whit the manuscript (see Additional file 1). 


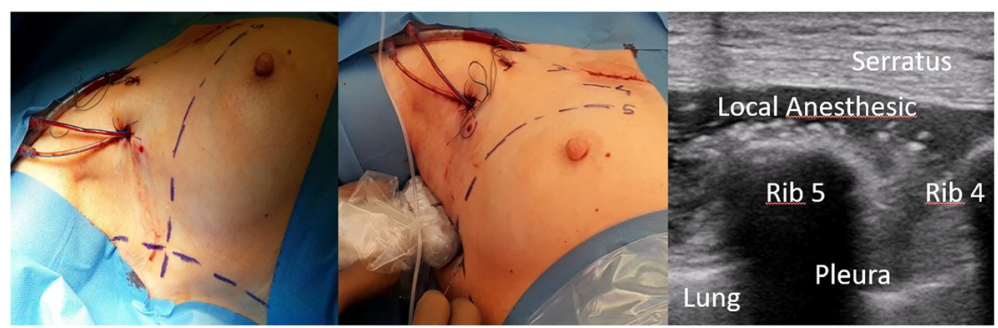

Fig. 1 SAPB landmarks, punction, and echographic view

\section{Statistical analysis}

Two groups of patients were compared: patient with CWI and patient with SAPB.

Quantitative data were expressed as means and standard deviations for variables with normal distribution and as medians and interquartile ranges (IQR) otherwise. Qualitative data were expressed as numbers and frequencies. Quantitative data are shown in box plots and were compared using the appropriate statistical test (Student's test or a non-parametric Mann-Whitney Wilcoxon test depending on the properties of the variable). Qualitative data were compared using the appropriate statistical test (Chi-2 with or without correction for continuity or Fisher's exact test depending on the properties of the variable).

A value $p \leq 0.05$ was considered significant for differences between the two groups. StataCorp 14.0 (Statacorp 4905 Lakeway Drive College Station, TX 77845 USA) was used for the statistical analysis.

\section{Results}

\section{Baseline characteristics}

During the observational period, 345 patients were screened in cardiac surgery, 48 patients have operated by MISH, and 2 patients were excluded for prolonged mechanical ventilation more than $24 \mathrm{~h}$ postoperatively, 1 for acute respiratory distress syndrome and one for hemorrhagic shock with surgical revision. We included 26 patients in CWI group and 20 SAPB.

The baseline characteristics are presented in Table 1. There were no significant baseline differences between the two groups.

\section{Primary outcome results}

The perioperative dose of sufentanil was comparable in the two groups with a median of $103 \mu \mathrm{g}$ [90-118] in the CWI group and $108 \mu \mathrm{g}$ [94-128] in the SABP group $(p=0.26)$. The median consumption of morphine $(\mathrm{mg})$ in the CWI and SAPB group was 21 versus $11 \mathrm{mg}$, respectively, $p<0.01$ (Table 2).

\section{Other results}

Morphine titration (mg at h0) was significantly lower in the SABP group (5 [4-8] vs. $2[0-4] ; p<0.01)$. The cumulative doses of morphine $(\mathrm{mg})$ were significantly lower in the SAPB group at h6 (10 [7-15] vs 7 [4-10]; $p=0.01)$, h24 (18 [10-31] vs 10 [6-13]; p < 0.01) (Fig. 2). The pain evaluated by NRS before morphine titration was significantly lower in the SABP group (5 [4-6] vs. 0 [0-4]; $\mathrm{p}<0.01$ ). During the first $6 \mathrm{~h}$ and from the 6th to the 24th hour the pain tended to be lower in the SABP group but the difference was not statistically significant (Table 2). The lengths of stay (in hours) in ICU (41 [25-69] vs 24 [20-41]; $p=0.03)$ and in the hospital (8 [7-9] vs. $6[5-8] ; p=0.03$ ) are significantly shorter in the SAPB group. There was no statistically significant difference in the incidence of nausea and vomiting outcomes between the groups $(p=0.7)$. The need of NIV was reduce in SAPB group, $15 \%$ versus $23 \%$ in CWI group, but the result was not significate $(p=0.7)$.

Table 1 General characteristics of the studied groups

\begin{tabular}{llll}
\hline & $\begin{array}{l}\text { CWI group } \\
(n=26)\end{array}$ & $\begin{array}{l}\text { SAPB group } \\
(n=20)\end{array}$ & $p$ Value \\
\hline Sex ratio (M/F) & $18 / 8$ & $10 / 10$ & 0.23 \\
Age & $67[64-72]$ & $67[60-74]$ & 0.78 \\
BMI (kg/m²) & $27[25-29]$ & $29[23-31]$ & 0.50 \\
Euroscore 2 & $1.2[0.8-2.0]$ & $0.9[0.7-1.6]$ & 0.20 \\
Respiratory disease & $4(15)$ & $4(20)$ & 0.70 \\
Characteristics of the surgery & & & \\
$\quad$ AVR & $21(81)$ & $14(70)$ & 0.49 \\
$\quad$ MVR & $4(15.4)$ & 0 & 0.12 \\
$\quad$ Mitral valve plasty & $1(3.9)$ & $5(25)$ & 0.07 \\
CABG & 0 & $1(5)$ & 0.46 \\
Duration of surgery (min) & $192[170-227]$ & $194[167-216]$ & 0.62 \\
Intraoperative data & & & \\
$\quad$ Gabapentin & $25(96)$ & $19(94)$ & 0.76 \\
Ketamine & $8(31)$ & $7(35)$ & 0.50 \\
Sufentanil ( $\mu$ g) & $103[90-118]$ & $108[94-128]$ & 0.26 \\
Duration anesthesia (h) & $10[9-11]$ & $9[9-10]$ & 0.48 \\
Time to extubation (h) & $5[4-6]$ & $5[4-6]$ & 0.90 \\
\hline
\end{tabular}

Date are presented as median [IQR] or $\mathrm{n}(\%)$

$A V R$ aortic valve replacement, $M V R$ mitral valve replacement, $C A B G$ coronary artery bypass graft surgery, Euroscore 2 European System for Cardiac Operative Risk Evaluation: risk model which allows the calculation of the risk of death after a heart operation 
Table 2 Morphine consumption, NRS and additionnal analgesics during the first $48 \mathrm{~h}$ in the two studied groups

\begin{tabular}{llll}
\hline & $\begin{array}{l}\text { CWl group } \\
(n=26)\end{array}$ & $\begin{array}{l}\text { SABP group } \\
(n=20)\end{array}$ & $p$ Value \\
\hline $\begin{array}{llll}\text { Dose of morphine }(\mathrm{mg}) \\
\text { Total to } 48 \mathrm{~h}\end{array}$ & $21[11-36]$ & $11[5-19]$ & $<0.01$ \\
Titration (h0) & $5[4-8]$ & $2[0-3.5]$ & $<0.01$ \\
h0-h6 & $10[7-15]$ & $7[4-10]$ & 0.01 \\
h0-h24 & $18[10-31]$ & $10[6-13]$ & $<0.01$ \\
NRS & & & \\
h0 & $5[4-6]$ & $0[0-4]$ & $<0.01$ \\
h6 & $2[0-4]$ & $0[0-3]$ & 0.13 \\
h24 & $0.5[0-3]$ & $0[0-2]$ & 0.41 \\
h48 & $0[0-2]$ & $0[0-2]$ & 0.85 \\
Additional analgesics & & & \\
Nefopam & $6(23)$ & $1(5)$ & 0.20 \\
Tramadol & $5(19)$ & $0(0)$ & 0.06 \\
Ketoprofen & $4(15)$ & $5(25)$ & 0.66 \\
Nausea and vomiting & $8(30)$ & $5(25)$ & 0.7 \\
NIV & $6(23)$ & $3(15)$ & 0.7 \\
Length of stay & & & 0.03 \\
ICU (h) & $41[25-69]$ & $24[20-42]$ & 0.03 \\
Hospital (days) & $8[7-9]$ & $6-8]$ & \\
\hline Data are presented as median [IQR] or $n$ (\%) \\
CWI catheter wound infiltration group, SAPB serratus anterior plane block group, \\
NRS visual analogic scale, NIV noninvasive ventilation, ICU intensive care unit
\end{tabular}

\section{Discussion}

Our study demonstrated that patients treated with SAPB had a significantly lower total morphine consumption during the first $48 \mathrm{~h}$ following minimally invasive heart surgery and lower NRS.

Analysis of morphine consumption in the different periods revealed that the difference was mainly related to a significantly lower dose of morphine titrated in SABP patients. In cumulative dose analysis, this difference persisted throughout the study period. This result was correlated with the pain experienced by the patient, because prior to morphine titration, NRS was significantly higher in the CWI group, accounting for the greater morphine consumption. Single-injection SAPB seems effective for early pain, but its effect is too short to provide adequate analgesia for $48 \mathrm{~h}$ after surgery. This result is in agreement with the study by Hetta et al., who reported a median duration of analgesia of six hours, and with the results of Blanco et al., who reported duration between 6 and $10 \mathrm{~h}[11,17]$. Median extubation time in our patients was $5 \mathrm{~h}$; the morphine titration was therefore given towards the end of the SAPB efficacy period, thus explaining the lower doses injected at that time and the absence of any significant difference at subsequent time points.

CWI in patients who underwent thoracotomy was also investigated in the literature, but does not lead to a decrease in morphine consumption compared to a PCA alone [18]. The metameric spread of SAPB was not

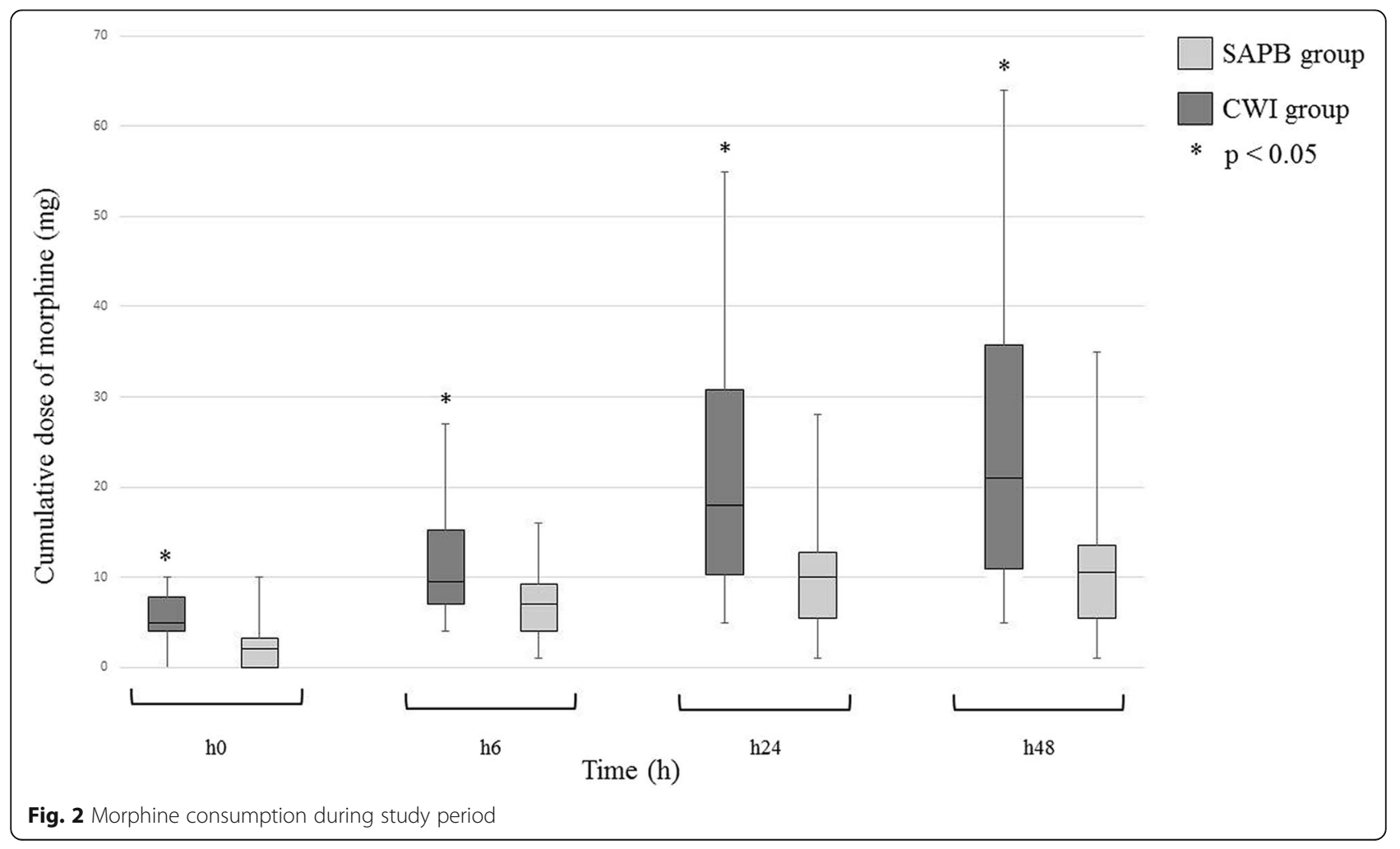


evaluated in our study; usually SAPB covers dermatomes T2 to T6, with variable degrees of cephalad spread reported in cadaveric studies $[19,20]$. Nonetheless, the the surgical incision in MIHS, is systematically covered in the metameric spread of the block. Effectiveness of SAPB was also shown in a randomized-controlled trial for patients who undergo breast surgery. The analgesic result achieved with SAPB was good, compared with para-vertebral block [17]. The efficacy of SAPB has also been evaluated in thoracotomy and thoracoscopic surgery, with a reduction in pain and opioid consumption [21,22].

Because our cohort was too small, we were unable to find a significant difference in nausea and vomiting and use of NIV. Okmen et al. did not demonstrate a difference for these criteria, but the number of patients was too small [22]. We can assume that painless patients had earlier post-operative rehabilitation with respiratory physiotherapy sessions in the first hours following extubation to reduce not only the respiratory morbidity associated with this type of intervention, but also the length of stay in the ICU and in hospital and thus the overall cost of care.

SAPB analgesia may have better hemodynamic stability compared to epidural analgesia, with no significant difference for NRS on the $24 \mathrm{~h}$ after surgery [23]. We did not observe any adverse events related to SAPB. This peripheral block seems easiest to apply and it is fast learning compared to perimedullary techniques. An experienced operator can perform the technique without assistance and without prolonging the overall duration of the anesthesia or the time between arrival in the ICU and extubation.

To overcome the short-lasting efficacy of SAPB, some authors have described the possibility of inserting a catheter into the SAPB space to allow continuous infusion of a local anesthetic for a predetermined time, after an initial bolus $[14,24,25]$. Such catheter insertion into the SAPB space for continuous ropivacaïne infusion could improve pain control during the first $48 \mathrm{~h}$ without opioid.

This retrospective study has several limitations. In particular, it's retrospective study, without randomization and without blindness. However, the small size is quite close to the numbers of prospective randomized studies on SAPB. In the absence of preliminary data, we designed a retrospective comparative (exploratory) study with a convenience sample of 48 consecutive patients.

\section{Conclusion}

SAPB may be effective in decreasing morphine consumption and pain after MIHS. A large double blind randomized trial is essential to confirm our results. Catheter insertion in the SAPB space with continuous infusion of a local anesthetic could be an interesting alternative for providing prolonged analgesia in the first days after surgery.

\section{Additional file}

Additional file 1: Data of SAPB versus CWI study. Additional file contains demographic data of patients, morphine consumption and post operative pain in the SABP and CWI groups. (XLSX $26 \mathrm{~kb}$ )

\begin{abstract}
Abbreviations
AVR: Aortic valve replacement; BMI: Body mass index; CABG: Coronary Artery Bypass Graft Surgery; CNIL: Commission national de l'informatique et des libertées; CWI: Continuous wound infiltration; ICU: Intensive care unit; IQR: Interquartile range; MIHS: Minimally-invasive heart surgery; MVR: Mitral valve replacement;; NIV: Non invasive ventilation; NMT: Neuromuscular transmission; NRS: Numeric rating scale; PCA: Patient controlled analgesia; SAPB: Serratus anterior plane block
\end{abstract}

\section{Acknowledgements}

None.

Funding

None to declare.

Availability of data and materials

Our data are available in Additional file 1.

Authors' contributions

VB, OE, MK, GM, SA and MN designed a retrospective study, and performed data collection and analysis. VB, OE and MN wrote manuscript. CG, PGG, OB and BB have reviewed manuscript. All authors read and approved the final manuscript.

Ethics approval and consent to participate

Consent to participate was waived due to retrospective character of the study. SABP and CWI were realized as routine professional practices in our center. Due to the use of patient data, we submitted our study to the national commission for data protection (CNIL) and obtained authorization which is $2053943 \vee 0$.

\section{Consent for publication}

NA

\section{Competing interests}

The authors declare that they have no competing interests.

\section{Publisher's Note}

Springer Nature remains neutral with regard to jurisdictional claims in published maps and institutional affiliations.

\section{Author details}

'Unité d'Anesthesie Réanimation Cardio-Vasculaire, CHU François Mitterrand, BP 77908, 21709 Dijon Cedex, France. 'Service d'Epidémiologie et d'Hygiène Hospitalières, CHU François Mitterrand, BP 77908, 21709 Dijon Cedex, France. ${ }^{3}$ Service de Chirurgie Cardiaque, Vasculaire et Thoracique, CHU François Mitterrand, BP 77908, 21709 Dijon Cedex, France.

Received: 2 June 2018 Accepted: 10 October 2018

Published online: 20 October 2018

References

1. Schmitto JD, Mokashi SA, Cohn LH. Minimally-invasive valve surgery. J Am Coll Cardiol. 2010;56(6):455-2.

2. Wenk M, Schug SA. Perioperative pain management after thoracotomy. Curr Opin Anaesthesiol. 2011;24(1):8-12.

3. Karmakar MK, Ho AMH. Postthoracotomy pain syndrome. Thorac Surg Clin. 2004;14(3):345-2.

4. Pisani MA, Murphy TE, Araujo KLB, et al. Benzodiazepine and opioid use and the duration of intensive care unit delirium in an older population. Crit Care Med janv. 2009;37(1):177-3.

5. Mantz J, Hemmings HC, Boddaert J. Case scenario: postoperative delirium in elderly surgical patients. Anesthesiology. 2010;112(1):189-5. 
6. Fletcher D, Aubrun F. Long texts for the formalized recommendation of experts on management of postoperative pain. Ann Fr Anesth Réanimation. 2009;28(1):1-2

7. Comité douleur-anesthésie locorégionale et le comité des référentiels de la Sfar. Formalized recommendations of experts 2008. Management of postoperative pain in adults and children. Ann Fr Anesth Réanimation. 2008; 27(12):1035-41.

8. Horlocker TT. Peripheral nerve blocks--regional anesthesia for the new millennium. Reg Anesth Pain Med. 1998;23(3):237-40.

9. Rosen DA, Hawkinberry DW, Rosen KR, et al. An epidural hematoma in an adolescent patient after cardiac surgery. Anesth Analg. 2004:98(4):966-9.

10. Gebhardt R, Mehran RJ, Soliz J, et al. Epidural versus ON-Q local anestheticinfiltrating catheter for post-thoracotomy pain control. J Cardiothorac Vasc Anesth. 2013;27(3):423-6.

11. Blanco R, Parras T, McDonnell JG, et al. Serratus plane block: a novel ultrasound-guided thoracic wall nerve block. Anaesthesia. 2013;68(11):1107-13.

12. Bhoi D, Pushparajan HK, Talawar $P$, et al. Serratus anterior plane block for breast surgery in a morbidly obese patient. J Clin Anesth. 2016;33:500-1.

13. Kunhabdulla NP, Agarwal A, Gaur A, et al. A. Serratus anterior plane block for multiple rib fractures. Pain Physician. 2014;17(5):651-3.

14. Ökmen K, Okmen BM, Uysal S. Serratus anterior plane (SAP) block used for thoracotomy analgesia: a case report. Korean J Pain. 2016;29(3):189-92.

15. Takimoto K, Nishijima K, Ono M. Serratus plane block for persistent pain after partial mastectomy and axillary node dissection. Pain Physician. 2016; 19(3):481-6.

16. Berthoud V, Ellouze O, Bièvre T, Konstantinou, et al. Serratus anterior plane block for apical TAVR in an awake patient. J Cardiothorac Vasc Anesth 2017; https://doi.org/10.1053/j.jvca.2017.12.042

17. Hetta DF, Rezk KM. Pectoralis-serratus interfascial plane block vs thoracic paravertebral block for unilateral radical mastectomy with axillary evacuation. J Clin Anesth. 2016;34:91-7.

18. Fortier S, Hanna HA, Bernard A, et al. Comparison between systemic analgesia, continuous wound catheter analgesia and continuous thoracic paravertebral block: a randomised, controlled trial of postthoracotomy pain management. Eur J Anaesthesiol. 2012;29(11):524-30

19. Daga V, Narayanan MK, Dedhia JD, Gaur P, et al. Cadaveric feasibility study on the use of ultrasound contrast to assess spread of injectate in the serratus anterior muscle plane. Saudi J Anaesth. 2016;10(2):198-201.

20. Mayes J, Davison E, Panahi P, Patten D, et al. An anatomical evaluation of the serratus anterior plane block. Anaesthesia. 2016;71(9):1064-9.

21. Ökmen K, Metin Ökmen B. Evaluation of the effect of serratus anterior plane block for pain treatment after video-assisted thoracoscopic surgery. Anaesth Crit Care Pain Med 2017; https:/doi.org/10.1016/j.accpm.2017.09.005.

22. Ökmen K, Ökmen BM. The efficacy of serratus anterior plane block in analgesia for thoracotomy: a retrospective study. J Anesth. 2017;31(4):579-85.

23. Khalil AE, Abdallah NM, Bashandy GM, et al. Ultrasound-guided serratus anterior plane block versus thoracic epidural analgesia for thoracotomy pain. J Cardiothorac Vasc Anesth. 2017:31(1):152-8.

24. Bossolasco M, Bernardi E, Fenoglio LM. Continuous serratus plane block in a patient with multiple rib fractures. J Clin Anesth. 2017:38:85-6.

25. Madabushi R, Tewari S, Gautam SK, et al. Serratus anterior plane block: a new analgesic technique for post-thoracotomy pain. Pain Physician. 2015; 18(3):E421-4.

Ready to submit your research? Choose BMC and benefit from:

- fast, convenient online submission

- thorough peer review by experienced researchers in your field

- rapid publication on acceptance

- support for research data, including large and complex data types

- gold Open Access which fosters wider collaboration and increased citations

- maximum visibility for your research: over $100 \mathrm{M}$ website views per year

At $\mathrm{BMC}$, research is always in progress.

Learn more biomedcentral.com/submissions 